\title{
Is This Designation Really Necessary? ${ }^{1}$
}

\author{
R. F. GRIFFIN \\ The Observatories, Madingley Road, Cambridge, CB3 OHA, UK
}

The only reason that comes to mind why 'visual' binaries (whether discovered visually or not) conventionally receive discoverers' designations, whereas spectroscopic ones do not, is that many visual binaries were discovered before most of the stars concerned had any other designations; spectroscopic binaries were not invented until after the B.D. was published. It is accordingly suggested, very gently, that it no longer serves a useful purpose to attach additional identification numbers to stars that are already well known, and it could even be argued that unnecessary proliferation of designations is best avoided.

${ }^{1}$ With acknowledgements to van den Bos (PASP, 74, 297, 1962) 